\title{
Adaptación cultural de la escala de resiliencia para niños en la Institución Educativa Primaria Bellavista de la UGEL Juliaca
}

\section{Cultural adaptation of the resilience scale for children in the Bellavista Elementary Educational Institution of UGEL Juliaca}

\author{
Verónica Pineda Alcos ${ }^{1}$
}

\begin{abstract}
RESUMEN
Objetivo: Realizar una adaptación cultural de la Escala Resiliencia para Jóvenes y Adultos a la una Escala de Resiliencia Escolar para niños de 10 a 12 años, que posea validez y confiabilidad, aplicado a niños del quinto y sexto grado de la Institución Educativa Primaria Bellavista de la ciudad de Juliaca, 2019. Metodología: La presente investigación corresponde a un enfoque cuantitativo, así mismo se utilizó un diseño no experimental de corte transversal y es de tipo psicométrico. La población abarca 168 estudiantes del quinto y sexto grado de la Institución Educativa Primaria Bellavista, cuyas edades oscilen entre los 10 a 12 años de edad. Para el análisis de datos se utilizaron los estadísticos de V de Aiken y alpha de cronbach. Resultados: Se obtuvo un coeficiente alfa de Cronbach de 0.732 lo cual indica que la escala es altamente confiable. Conclusión: La adaptación cultural de la escala de resiliencia para niños evidencia buena consistencia interna y validez de constructo.
\end{abstract}

Palabras clave: Resiliencia, validez, confiabilidad, niños, resiliencia escolar.

\begin{abstract}
Objective: To carry out a cultural adaptation of the Resilience Scale for Young People and Adults to a School Resilience Scale for children from 10 to 12 years old, which has validity and reliability, applied to children of the fifth and sixth grade of the Bellavista Elementary Educational Institution of the city of Juliaca, 2019. Methodology: This research corresponds to a quantitative approach, likewise a nonexperimental cross-sectional design was used and it is of a psychometric type. The population includes 168 fifth and sixth grade students from the Bellavista Elementary Educational Institution, whose ages range from 10 to 12 years old. For data analysis, Aiken's $V$ statistics and Cronbach's alpha were used. Results: Cronbach's alpha coefficient of 0.732 was obtained, which indicates that the scale is highly reliable. Conclusion: The cultural adaptation of the resilience scale for children shows good internal consistency and construct validity.
\end{abstract}

Keywords: Resilience, validity, reliability, children, school resilience. 


\section{INTRODUCCIÓN}

En la actualidad el termino resiliencia es de suma importancia en el ámbito pedagógico, ya que en las escuelas se reciben a diferentes alumnos los cuales algunos están en situación de desventaja social, familiar o personal, por lo tanto, suelen ser sujetos de exclusión educativa como el fracaso escolar, la inadaptación social y conflictos escolares. Así que es necesario que estos estudiantes puedan afrontar los nuevos retos de la educación actual, de la misma forma deben buscar la forma de desarrollarse como personas capaces de participar activamente en la sociedad y afrontar con éxito las inevitables dificultades de la vida. El tema de la resiliencia está dejando de ser un tema emergente para convertirse en un tema central en los campos de la psicología y específicamente en la psicología positiva, así como también es un tema muy importante para la pedagogía (Aguilar, Arjona, \& Noriega, 2015).

Diversos estudios han destacado el papel de la escuela, el profesor y las experiencias escolares en la construcción de la resiliencia. La escuela puede ser un contexto para el desarrollo integral y para la resiliencia de todos los alumnos, aparte de la función cognoscitiva que tiene de enseñar y aprender es un espacio de comunicación, da oportunidades a sus alumnos para establecer vínculos positivos que en algunos casos compensen experiencias negativas de otros contextos sociales (Uriarte, 2006).

En Chile, Jadue, Galindo y Navarro, en el 2005 realizaron un estudio cualitativo de tipo descriptivo con el propósito de analizar los factores protectores y factores de riesgo para el desarrollo de la resiliencia encontrados en una comunidad educativa de riesgo para el desarrollo educativo de riesgo social, en una muestra de 192 participantes entre niños con sus respectivos padres y docentes, a los que se aplicó encuestas descriptivas, entrevistas individuales, Inventario de Autoestima de Coopersmith; técnicas como observación participante, revisión y análisis de fuentes directas. Los hallazgos que encontraron es que la docencia presenta un bajo nivel en su experiencia profesional. Por lo que concluyen que es necesario trabajar con las potencialidades y recursos que los integrantes de la comunidad educativa poseen, mejorando la relación de la comunidad educativa, contribuyendo al cambio de mentalidad de los docentes hacia sus alumnos y familias.
Así mismo, en el Perú, Silva (2012), realizo una investigación a fin de determinar el nivel de resiliencia de los alumnos de 9 a 12 años, de un colegio. La muestra fue de 150 alumnos. El tipo de investigación fue cuantitativa, utilizaron el instrumento de factores personales de resiliencia creado por Salgado (2005). Los resultados determinaron un nivel medio de resiliencia en los estudiantes, lo que significa que se encuentran en proceso de mejorar sus capacidades y habilidades para sobreponerse a la adversidad. El autor concluyó que los factores de resiliencia a excepción de autoestima y empatía (nivel alto) se encuentran en el nivel medio.

En Juliaca, Huayto y Leonardo (2016) ejecutaron una investigación titulada "Clima social familiar y resiliencia en estudiantes del cuarto y quinto grado de secundaria del Colegio Adventista Túpac Amaru de la ciudad de Juliaca - 2015". Esta investigación fue de un diseño de corte transversal no experimental de tipo descriptivo correlacional, para la recolección de datos se utilizaron las escala del Clima social Familiar (FES) y la escala para adolescentes (ERA) la población estuvo conformado por 201 estudiantes del cuarto y quinto año de secundaria donde se obtuvo una muestra de 92 estudiantes seleccionado mediante un muestreo probabilístico estratificado, hallándose a si una correlación directa y significativa entre el Clima social familiar y Resiliencia $(r=, 534 ; p<0.05)$.

Noriega, Angulo y Angulo (2015) en su artículo la resiliencia en la educación, escuela y vida destacaron el papel de la escuela, el profesor y las experiencias escolares en la construcción de la resiliencia. La escuela puede ser un contexto para el desarrollo integral y para la resiliencia de todos los alumnos, aparte de la función cognoscitiva que tiene de enseñar y aprender es un espacio de comunicación, da oportunidades a sus alumnos para establecer vínculos positivos que en algunos casos compensen experiencias negativas de otros contextos sociales (Uriarte, 2006).

El concepto de resiliencia es usado cada vez más, tanto en educación, salud y ciencias sociales en general. Diversas son las definiciones de este fenómeno y distintas aproximaciones al estudio de ésta, se han realizado en las últimas dos décadas. No cabe duda que esta divulgación del tema ha despertado un alto interés tanto en estudiantes de educación superior, como en profesionales, por llevar a cabo estudios e 
investigaciones que nos acerquen a una mayor comprensión de este concepto. Gran parte de sus necesidades cognitivas, emocionales y relacionales las encuentran en la escuela un medio para satisfacerlas. Desde hace tiempo la sociedad conoce que las emociones y las relaciones sociales influyen en los procesos de enseñanza-aprendizaje y encarga a la escuela que, además de los objetivos cognoscitivos e instrumentales, también los aspectos del desarrollo socio afectivo se conviertan en objetivos educativos explícitos.

El término resiliencia cuyo origen procede del verbo latino resilio, proviene de la ingeniería basado en la mecánica, donde se hace mención a las características que presentan ciertos materiales al momento de ser empleados en la construcción, pues pueden recobrar la forma original con la que fueron creados después de haber sido expuestos a una presión deformadora (Gianino, 2012).

El término resiliencia define la capacidad que tienen las personas para desarrollarse psicológicamente con normalidad, a pesar de vivir en contextos de riesgo, como entornos de pobreza y familias multiproblemáticas, situaciones de estrés prolongado, centros de internamiento, etc. Se refiere tanto a los individuos en particular como a los grupos familiares o escolares que son capaces de minimizar y sobreponerse a los efectos nocivos de las adversidades y los contextos desfavorecidos socioculturalmente (Uriarte, 2005).

Ante múltiples definiciones de Resiliencia, se puede concluir que es el resultado de la interacción entre las cualidades de la persona (características internas) y su ambiente familiar, social y cultural (características externas) que lo facultan para superar el riesgo y la adversidad de forma constructiva (González, 2018).

El fundamento teórico que se está utilizando en la presente investigación es la perspectiva de Edith Grotberg que ubica al lenguaje como constructor de la realidad. En tal sentido, la resiliencia es expresión humana de generar realidades que posibilitan el desarrollo. Por otra parte, la perspectiva de Saavedra (2003) recoge el carácter histórico de la constitución de la resiliencia proyectando sus posibilidades más allá de la acción y sus resultados para postular la reorganización de los diversos factores que describen los diversos estudios, en ámbitos de menor a mayor profundidad en la reflexión de la acción. Es interesante notar el carácter complementario de la perspectiva de Grotberg y Saavedra en el sentido de abordar los diversos niveles de la realidad del sujeto que contribuyen a la constitución de la resiliencia y las modalidades interactivas de su manifestación en el habla. Es decir, cuando el sujeto habla de si, expresa, a propósito de su historia, una interpretación de sí, de los otros y lo que posee para abordar las situaciones actuales de su vida; es decir las categorías de Grotberg.

Grotberg (1995) menciona las características o factores que intervienen en los procesos resiliente que tienen que ver con la manera como el sujeto ubica el lenguaje, como constructor de la realidad y a su vez abre la posibilidad al comportamiento saludable o de superación de los eventos traumáticos creando así sus 3 fuentes de resiliencia. En primer lugar, el individuo cuenta con una o más personas dentro o fuera del entorno familiar con las que pueden confiar, estas le brindan estabilidad y lo alientan a ser independiente, así como, establecer límites, a este aspecto se le denomina el YO TENGO. En segundo lugar, abarca los recursos personales de la persona que comprende desde métodos de planificación y expectativas favorables hacia el futuro, asimismo las relaciones y establecimientos vínculos, poder identificarse con los sentimientos de los demás comprende el aspecto de YO SOY/YO ESTOY. Por último, el YO PUEDO se entiende como las habilidades y estrategias de afrontamiento.

Por tal contábamos con la dificultad de no contar con instrumentos válidos y confiables, que midan esta dimensión y que estén probados en nuestra población nacional, se sabe que algunas de ellas presentan una adecuada calidad, pero no responden a distintos patrones culturales, debiendo adaptar el lenguaje y el sentido de los textos. La necesidad de contar con un instrumento nacional, confiable y válido, que mida resiliencia para el segmento escolar, nos lleva hoy día a presentarles una escala que abarca entre los 10 y 12 años de edad y que está basada en la estructura y contenido del SV-RES. Esperamos que este instrumento signifique un aporte a quienes estudian esta dimensión en los niños y para quienes hacen esfuerzos por desarrollar conductas resilientes a nivel escolar, motivo que nos llevó a desarrollar la presente adaptación y así poder realizar un aporte a nuestra ciencia y la psicología. 


\section{METODOLOGÍA}

El presente trabajo de investigación corresponde a un tipo cuantitativo, puesto que se anhela identificar el comportamiento de una variable en poblaciones con diferentes características de estudio, el diseño tiene características acordes con el diseño No experimental, es decir, no se pretende manipular una variable, esta se evaluará en su ambiente natural, además posee una clasificación transaccional, porque se recogerán datos en un solo punto de tiempo (Hernandez, Fernandez, \& Baptista, 2014).

\section{Participantes}

Para la selección de los participantes se empleó el método no probabilístico por conveniencia, de esta manera se contó con un total de 168 estudiantes de quinto y sexto grado de primaria de la Institución Educativa Primaria BellavistaJuliaca. La población de estudio posee edades que oscilan entre los 10 a 12 años (donde la edad de 12 años es la que se repite más), así mismo, el género más numeroso fue el femenino con 89 mujeres, siendo el $53 \%$ y 79 de género masculino, siendo el $47 \%$; por otro lado, su lugar de procedencia el $100 \%$ proviene de la sierra; en cuanto a los grados 54 pertenecen al 5 to grado siendo el $32.1 \%$ y 114 pertenecen al 6 to grado siendo el $67.9 \%$; finalmente el $61.9 \%$ es de religión católica, el $14.9 \%$ de religión adventista, el $7.1 \%$ de religión evangélico y el $16.1 \%$ conciernen a otras religiones.

\section{Instrumentos}

El Instrumento que se utilizó para la validación fue la escala de resiliencia para jóvenes y adultos. Esta escala de origen Chileno fue creada por el Dr. Eugenio Saavedra Guajardo y el Dr. Marco Villalta Paucar en el año 2008, para identificar la existencia de resiliencia en su cultura; la escala original está conformada por 60 ítems compuestos por la escala Likert con 5 elecciones de frecuencia.

Por lo que fue adaptada a una escala de resiliencia para niños, la cual está constituida por 27 ítems, con respuestas de tipo likert, los cuales son Nunca, Casi Nunca, A Veces Casi Siempre y Siempre a través de cinco dimensiones mediante los cuales se pueden identificar los niveles de resiliencia escolar (alto, medio, bajo).

\section{Análisis de datos}

Los investigadores comenzaron con la revisión exhaustiva de la literatura para hacer la matriz instrumental a partir de un problema social significativo, después de hacer la matriz instrumental el cuestionario adaptado pasó a ser evaluado por 3 jueces bajo 4 criterios (claridad, congruencia, dominio del constructo y contexto) todos ellos dieron un veredicto final. así, mismo se procedió a aplicar una prueba piloto de 18 personas, el alfa de crombach este nos permitió sacar un resultado para después aplicar a una población más grande.

\section{RESULTADOS}

En la tabla 1 se observa el Alpha de Cronbach que debería ser utilizado en escalas Likert como la que presentamos en esta investigación, el índice de confiabilidad o consistencia interna alcanza un 0.732 , esto indica que el instrumento es altamente confiable.

Tabla 1

Alfa de crombach de la escala de resiliencia escolar

\begin{tabular}{ll} 
Alfa de Cronbach & N de elementos \\
, 732 & 27 \\
\hline
\end{tabular}

En la tabla de 2 se observa los resultados de la validez de la prueba por $v$ de aiken por criterio de jueces, que permite señalar que en claridad tenemos un resultado de 0.88 , en congruencia un resultado de 0.92 , en contexto un resultado de 0.93 y en dominio del constructo un resultado de 0.88 , lo que en total nos da un resultado de $\checkmark$ de Aiken de 0.90 lo cual indica que es válido.

Tabla 2

$\checkmark$ de Aiken de la escala de resiliencia escolar

\begin{tabular}{ll} 
Criterios & \\
Claridad & 0.88 \\
Congruencia & 0.92 \\
Contexto & 0.93 \\
Dominio del contructo & 0.88 \\
Total & 0.90 \\
\hline
\end{tabular}


En la tabla 3 se muestra resultados del análisis factorial confirmatorio, obtenidos mediante el método de máxima verosimilitud, con un supuesto de cinco factores, efectuando la prueba de KMO y Bartlett, donde los resultados estadísticos muestran un valor de KMO de 0.74 la cual recae dentro de la categoría de aceptable en calidad de valor, un Chi cuadrado de 883.02 y un P-valor (sig) que es significativo a nivel estadístico $(p=.000)$, lo que permite continuar con un análisis factorial confirmatorio.

Tabla 3

Prueba KMO y esfericidad de Bartlett

\section{Prueba de KMO y Bartlett}

Medida Kaiser-Meyer-Olkin de adecuación de muestreo

Prueba de esfericidad de Bartlett

Aprox. Chi-cuadrado

883,027

gl

351

Sig.

, 000

En la tabla 4 se observa la varianza total explicada, donde se obtiene 5 factores correlacionados por determinadas características en común, sin embargo, se debe considerar la cantidad de ítems pertenecientes a cada factor, para ser denominada como dimensión, entonces se obtiene como primeros resultados máximo a la varianza acumulada del factor 5 siendo explicado con el $40.97 \%$ del total.

Tabla 4

Varianza total explicada

\begin{tabular}{|c|c|c|c|c|c|c|}
\hline \multirow[b]{2}{*}{ Factor } & \multicolumn{3}{|c|}{ Autovalores iniciales } & \multicolumn{3}{|c|}{ Sumas de rotación de cargas al cuadrado } \\
\hline & Total & $\%$ de varianza & $\%$ acumulado & Total & $\%$ de varianza & $\%$ acumulado \\
\hline 1 & 4,899 & 18,145 & 18,145 & 1,859 & 6,884 & 6,884 \\
\hline 2 & 1,737 & 6,432 & 24,577 & 1,605 & 5,944 & 12,828 \\
\hline 3 & 1,570 & 5,815 & 30,392 & 1,498 & 5,548 & 18,376 \\
\hline 4 & 1,474 & 5,459 & 35,851 & 1,417 & 5,249 & 23,626 \\
\hline 5 & 1,382 & 5,119 & 40,970 & 1,379 & 5,107 & 28,732 \\
\hline 6 & 1,278 & 4,733 & 45,703 & & & \\
\hline 7 & 1,192 & 4,417 & 50,120 & & & \\
\hline 8 & 1,142 & 4,231 & 54,350 & & & \\
\hline 9 & 1,078 & 3,994 & 58,344 & & & \\
\hline 10 & 1,044 & 3,866 & 62,210 & & & \\
\hline 11 & ,957 & 3,546 & 65,756 & & & \\
\hline 12 & ,916 & 3,394 & 69,150 & & & \\
\hline 13 &, 875 & 3,242 & 72,391 & & & \\
\hline 14 & ,782 & 2,897 & 75,289 & & & \\
\hline 15 & ,756 & 2,799 & 78,087 & & & \\
\hline 16 & ,701 & 2,597 & 80,684 & & & \\
\hline 17 & ,635 & 2,351 & 83,035 & & & \\
\hline 18 &, 580 & 2,148 & 85,183 & & & \\
\hline 19 &, 556 & 2,059 & 87,242 & & & \\
\hline 20 &, 538 & 1,994 & 89,237 & & & \\
\hline
\end{tabular}




\begin{tabular}{lllll}
\hline & \multicolumn{2}{l}{ Autovalores iniciales } & \multicolumn{2}{c}{ Sumas de rotación de cargas al cuadrado } \\
Factor & Total & $\%$ de varianza & $\%$ acumulado & Total $\%$ de varianza \% acumulado \\
\hline 22 &, 469 & 1,737 & 92,903 & \\
23 &, 459 & 1,702 & 94,605 & \\
24 &, 411 & 1,524 & 96,128 & \\
25 &, 378 & 1,399 & 97,527 & \\
26 &, 345 & 1,278 & 98,805 & \\
27 &, 323 & 1,195 & 100,000 & \\
\hline
\end{tabular}

En la tabla 5 se observa la distribución y agrupación de los ítems en 5 factores. En su mayoría con cargas factoriales superiores a .20, caso contrario se descartó los ítems cuyos valores sean menores. Obteniendo como resultado 27 ítems validos en relación a la variable resiliencia.

Tabla 5

Matriz de factor rotado

\begin{tabular}{|c|c|c|c|c|c|}
\hline & $\begin{array}{l}\text { Identidad - } \\
\text { Autoestima } \\
1\end{array}$ & $\begin{array}{l}\text { Redes - } \\
\text { Modelo } \\
2\end{array}$ & $\begin{array}{l}\text { Aprendizaje - } \\
\text { Generatividad } \\
3\end{array}$ & $\begin{array}{l}\text { Recursos } \\
\text { internos } \\
4\end{array}$ & $\begin{array}{l}\text { Recursos } \\
\text { externos } \\
5\end{array}$ \\
\hline ITEM25 & ,502 & & & & \\
\hline ITEM24 & ,467 & & & & \\
\hline ITEM20 & ,456 & & & & \\
\hline ITEM2 & ,437 & & & & \\
\hline ITEM16 & 291 & & & & \\
\hline ITEM8 & ,289 & & & & \\
\hline ITEM19 & ,289 & & & & \\
\hline ITEM21 & ,267 & & & & \\
\hline ITEM18 & & ,461 & & & \\
\hline ITEM1 & & ,458 & & & \\
\hline ITEM11 & & ,444 & & & \\
\hline ITEM5 & &, 360 & & & \\
\hline ITEM9 & & ,342 & & & \\
\hline ITEM4 & & ,338 & & & \\
\hline ITEM26 & & ,304 & & & \\
\hline ITEM15 & & ,259 & & & \\
\hline ITEM14 & & ,244 & & & \\
\hline ITEM12 & & & ,712 & & \\
\hline ITEM13 & & & ,449 & & \\
\hline ITEM10 & & & ,441 & & \\
\hline ITEM23 & & & & ,688 & \\
\hline ITEM22 & & & & ,440 & \\
\hline ITEM3 & & & & ,418 & \\
\hline ITEM27 & & & & ,276 & \\
\hline ITEM17 & & & & &,- 815 \\
\hline ITEM6 & & & & & ,380 \\
\hline ITEM7 & & & & & ,376 \\
\hline
\end{tabular}


En la tabla 6 se ilustran los estadísticos totales por ítem cuyos índices de Alpha de Cronbach, si el elemento se elimina están por debajo del Alpha de Cronbach total, esto indicaría que ningún ítem debe ser eliminado.

Tabla 6

Estadísticos totales por ítem

\begin{tabular}{|c|c|c|c|c|}
\hline & $\begin{array}{l}\text { Media de escala si } \\
\text { el elemento se ha } \\
\text { suprimido }\end{array}$ & $\begin{array}{l}\text { Varianza de escala } \\
\text { si el elemento se ha } \\
\text { suprimido }\end{array}$ & $\begin{array}{l}\text { Correlación total } \\
\text { de elementos } \\
\text { corregida }\end{array}$ & $\begin{array}{l}\text { Alfa de Cronbach si } \\
\text { el elemento se ha } \\
\text { suprimido }\end{array}$ \\
\hline ITEM1 & 105,1488 & 143,984 & ,405 & ,719 \\
\hline ITEM2 & 105,6250 & 146,224 & ,198 & ,729 \\
\hline ITEM3 & 105,2321 & 147,257 & ,223 &, 727 \\
\hline ITEM4 & 105,4107 & 142,028 & ,352 & ,719 \\
\hline ITEM5 & 106,5238 & 142,862 & ,267 & ,724 \\
\hline ITEM6 & 105,0536 & 149,057 & ,143 & ,731 \\
\hline ITEM7 & 104,9940 & 145,216 & ,360 & ,722 \\
\hline ITEM8 & 105,9940 & 142,521 & ,334 &, 721 \\
\hline ITEM9 & 105,5238 & 146,107 & ,261 & ,725 \\
\hline ITEM10 & 104,9464 & 146,590 & ,292 & ,725 \\
\hline ITEM11 & 105,4940 & 144,443 & ,251 & ,725 \\
\hline ITEM12 & 105,1488 & 139,121 &, 511 & ,711 \\
\hline ITEM13 & 105,7381 & 138,626 & ,372 & ,717 \\
\hline ITEM14 & 105,9643 & 140,274 & ,344 & ,719 \\
\hline ITEM15 & 105,0060 & 147,144 & ,226 & ,727 \\
\hline ITEM16 & 104,7500 & 150,596 & ,183 & ,730 \\
\hline ITEM17 & 104,7738 & 142,404 &,- 037 & ,813 \\
\hline ITEM18 & 105,9405 & 140,655 & ,424 & ,715 \\
\hline ITEM19 & 106,6310 & 143,097 & ,254 & ,725 \\
\hline ITEM20 & 105,4524 & 141,016 & ,405 & ,717 \\
\hline ITEM21 & 106,6726 & 143,216 & ,259 & ,725 \\
\hline ITEM22 & 105,3869 & 142,358 & ,349 & ,720 \\
\hline ITEM23 & 105,3631 & 143,778 & ,348 & ,721 \\
\hline ITEM24 & 105,4643 & 137,400 &, 549 & ,708 \\
\hline ITEM25 & 105,7619 & 139,548 & ,452 &, 713 \\
\hline ITEM26 & 105,4226 & 141,766 & ,397 & ,717 \\
\hline ITEM27 & 104,8155 & 147,744 & ,334 & ,725 \\
\hline
\end{tabular}

En el gráfico 1 se observa la ilustración sedimentaria de los factores, lo que indica que a partir del factor 4 hay una continuidad uniforme, esto confirma la existencia de 5 factores en el instrumento. 


\section{Gráfico 1}

Gráfico de sedimentación

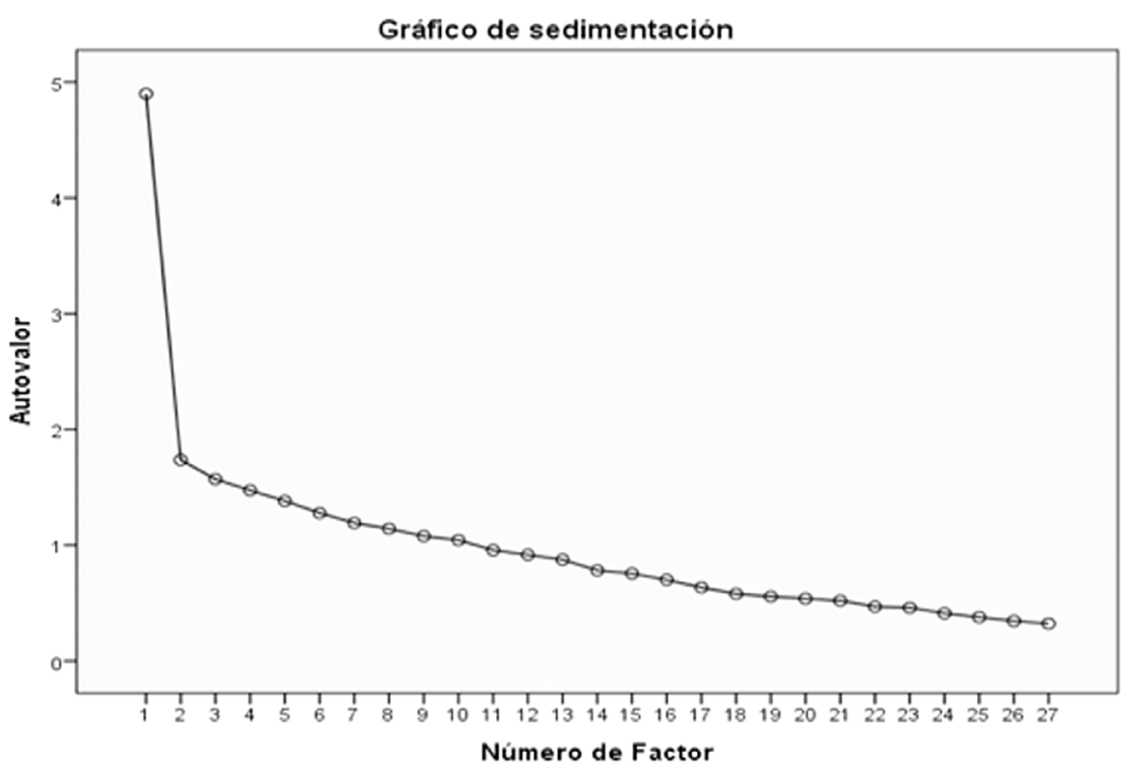

Tabla 7

Ítems de la escala de la escala de resiliencia para niños

\section{ITEMS}

1.- Yo soy una persona satisfecha de mí mismo.

2.- Yo soy optimista respecto del futuro.

3.- Yo estoy seguro de mí mismo.

4.- Yo me siento seguro en el ambiente en que vivo.

5.- Yo soy un modelo positivo para mis compañeros.

6.- Yo estoy satisfecho con mis amistades.

7.- Yo soy una persona con metas en la vida.

8.- Yo soy independiente.

9.- Yo soy responsable.

10.- Yo tengo una familia que me apoya.

11.- Yo tengo personas a quien recurrir en caso de problemas.

12.- Yo tengo personas que me orientan y aconsejan.

13.- Yo tengo personas que les puedo contar mis problemas.

14.- Yo tengo amigos que me cuentan sus problemas.

15.- Yo tengo buena relación con muchos profesores.

16.- Yo tengo proyectos a futuro

17.- Yo tengo en general una vida feliz

18.- Yo puedo en general resolver mis problemas.

19.- Yo puedo hablar de mis sentimientos con claridad ante los demás.

20.- Yo puedo expresar cariño.

21.- Yo puedo confiar en otras personas.

22.- Yo puedo dar mi opinión. 


\section{ITEMS}

23.- Yo puedo buscar ayuda cuando la necesito.

24.- Yo puedo apoyar a otros que tienen problemas.

25.- Yo puedo comunicarme bien con otras personas.

26.- Yo puedo aprender de mis aciertos y errores.

27.- Yo puedo esforzarme por lograr mis objetivos.

\section{DISCUSIÓN}

Paralapresenteinvestigaciónsebuscódesarrollar y evaluar las propiedades psicométricas (Validez de contenido, de constructo y confiabilidad) que siguieron un complejo procedimiento para demostrar evidencias científicas de la utilidad del instrumento, motivo por el cual se realizará la discusión con respecto a los resultados obtenidos en las investigaciones presentadas anteriormente y la presente.

En la investigación realizada en Chile por Villalta. (2008) donde se realizó la adaptación y traducción de la escala de resiliencia para jóvenes y adultos de 15 años a 65 años de edad. El cual ese puede aplicar de manera colectiva e individual, al igual que a ambos sexos.

KMO de (.59) y un resultado de Chi cuadrado de 2331, en cambio en el estudio de Mesías (2017) se obtuvo un puntaje en la prueba de KMO (.77) y un resultado de Chi cuadrado de 3634 , consideramos que el nivel obtenido en ambas investigaciones difiere a causa de la diferencia del idioma y el conocimiento de los términos por parte de la población evaluada en la presente investigación, ya que estos pueden variar según el lugar geográfico de estudio.

Como pudimos observar en las investigaciones realizadas por Verduzco, et al. (1994) y Mesías (2017), las cuales tienen amplia similitud con la presente investigación, se pudo identificar que al obtener los resultados del procedimiento estadístico de Análisis Factorial, este resulta en diferentes agrupaciones de factores, excediendo en más de 10 factores a las dimensiones originales, el motivo principal de estos resultados que parecen a primera impresión incoherentes o errados, es la diferencia del contexto social y en el presente estudio, una de las primeras aplicaciones de pruebas psicométricas en lengua nativa, tal como es el quechua.
Según los resultados obtenidos durante el transcurso del procesamiento de datos de la Escala de Resiliencia Escolar para niños de 10 a 12 años, se concluye:

Se logró adaptar culturalmente la Escala de Resiliencia para jóvenes y adultos "SV-Res" a la Escala de Resiliencia Escolar "PINZEL" para niños de 10 a 12 años, así mismo se logró desarrollar y medir sus propiedades psicométricas en una muestra de 168 niños entre 10 a 12 años de edad en la Institución Educativa Primaria Bellavista de la UGEL de la ciudad de Juliaca.

Para la validación psicométrica se utilizó el análisis factorial exploratorio en la Escala de Resiliencia Escolar "PINZEL", generando un KMO de 0.74 lo cual está dentro de los parámetros de la categoría "aceptable" en calidad de valor, considerando que el nivel de significancia es óptimo dentro de un análisis factorial exploratorio y confirmatorio.

\section{Correspondencia}

Verónica Pineda Alcos

Correo electrónico:

veronica.pineda@upeu.edu.pe 


\section{REFERENCIAS BIBLIOGRÁFICAS}

Aguilar, G., Arjona, B., \& Noriega, G. (2015). La resiliencia en la educación, la escuela y la vida. La resiliencia en la educación, la escuela y la vida, 58, 42-48. Recuperado de http://www. revistas.ujat.mx/index.php/perspectivas/article/ view/1199/974

Gianino, L. (2012). La resiliencia en niños institucionalizados y no institucionalizados. Av.psicol, 20(2), 2012.

González, N. (2018). Autoestima, Optimismo y Resiliencia en Niños en Situación de Pobreza. Revista Internacional de Psicología, 16(01), 1-119. https://doi.org/10.33670/18181023. v16i01.261

Grotberg, E (1995). A guide to promoting resilience in children: Strengthening the human spirit. The international resilience project, (8). Recuperado de https://bibalex.org/baifa/Attachment/ Documents/115519.pdf.

Grotberg, E. H. (2003). Resilience for Today: Gaining Strength from Adversity. Greenwood, SC: Praeger Publishers

Hernandez, R., Fernandez, C., \& Baptista, P. (2014). metodologia de la investigacion.

Huayto, Y., \& Leonardo, R. (2016). Clima social familiar y resiliencia en estudiantes del cuarto y quinto grado de secundaria del Colegio Adventista Túpac Amaru de la ciudad de Juliaca - 2015., 117. Recuperado de http://repositorio.upeu.edu. pe/bitstream/handle/UPEU/571/Yovana_Tesis_ bachiller_2016.pdf?sequence=1\&isAllowed=y

Jadue, G., Galindo, A., \& Navarro, L. (2005). FACTORES PROTECTORES Y FACTORES DE RIESGO PARA EL DESARROLLO DE LA RESILIENCIA ENCONTRADOS EN UNA COMUNIDAD EDUCATIVA EN RIESGO SOCIAL. Estudios Pedagógicos, XXXI (2), 43-55. https://doi.org/https://www.redalyc.org/ pdf/1735/173519073003.pdf

Noriega, G., Angulo, B., \& Angulo, G. (2015). La resiliencia en la educación, la escuela y la vida. La resiliencia en la educación, la escuela y la vida, 58, 42-48. Recuperado de http://www. revistas.ujat.mx/index.php/perspectivas/article/ view/1199/974
Saavedra, E. (2003) El Enfoque Cognitivo Procesal Sistémico, como posibilidad de Intervenir educativamente en la formación de sujetos Resilientes. Universidad de Valladolid, España.

Saavedra, E. y Villalta, M. (2008b). Escala de resiliencia SV-RES para jóvenes y adultos. Ceanim: Chile.

Saavedra, E. (2003) La Emoción como Construcción de Significados. Patio, Revista Pedagógica. № 27 Agosto - Octubre. ISSN 1518- 305X, Brasil.

Silva, R. (2012). Resiliencia en Estudiantes del V ciclo de Educación Primaria de una Institución del Callao. Resiliencia en estudiantes del V ciclio de Educación Primariadeunalnstitucióneducativadel Callao, 1, 1-115. Recuperado de http://repositorio. usil.edu.pe/bitstream/123456789/1327/1/2012 Silva_Resiliencia en estudiantes del V ciclo de educación primaria de una institución educativo del Callao.pdf

Uriarte, J. (2005). La resiliencia. Una nueva perspectiva en psicopatología del desarrollo Resilience. Revista de Psicodidáctica, 10(2), 61-79. Recuperado de https://www.redalyc.org/ pdf/175/17510206.pdf

Uriarte, J. (2006). Construir la resiliencia en la escuela. British Journal of School Nursing, 11(2), 7-24. https://doi.org/10.12968/bjsn.2017.12.2.98

Recibido: 20/03/2021

Aceptado: 23/05/2021 